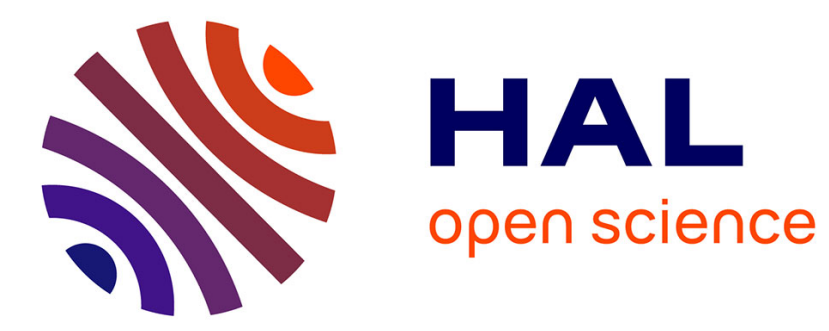

\title{
On the Moduli Space of Real Enriques Surfaces.
}

Alexander Degtyarev, Viatcheslav Kharlamov

\section{To cite this version:}

Alexander Degtyarev, Viatcheslav Kharlamov. On the Moduli Space of Real Enriques Surfaces.. 1996. hal-00129544

\section{HAL Id: hal-00129544 \\ https://hal.science/hal-00129544}

Preprint submitted on 8 Feb 2007

HAL is a multi-disciplinary open access archive for the deposit and dissemination of scientific research documents, whether they are published or not. The documents may come from teaching and research institutions in France or abroad, or from public or private research centers.
L'archive ouverte pluridisciplinaire HAL, est destinée au dépôt et à la diffusion de documents scientifiques de niveau recherche, publiés ou non, émanant des établissements d'enseignement et de recherche français ou étrangers, des laboratoires publics ou privés. 


\title{
On the moduli space of real Enriques surfaces
}

\author{
Alexander DEGTYAREV and Viatcheslav KHARLAMOV \\ A. D.: Steklov Mathematical Institute, St. Petersburg branch, 191011, St. Petersburg, Russia and \\ Bilkent University, Ankara, Turkey. E-mail: degt@ math.ucr.edu, degt@pdmi.ras.ru; \\ V. K.: Institut de Recherche Mathématique Avancée, Université Louis Pasteur et CNRS, \\ 7 rue René-Descartes, 67084, Strasbourg, France. E-mail: kharlam @ math.u-strasbg.fr.
}

\begin{abstract}
We introduce a new invariant, Pontrjagin-Viro form, of real algebraic surfaces. We evaluate it for real Enriques surfaces with non-negative minimal Euler characteristic of the components of the real part and prove that, when combined with the known topological invariants, it distinguishes the deformation types of such surfaces.
\end{abstract}

\section{Sur l'espace de modules des surfaces d'Enriques réelles}

Résumé. On introduit un nouvel invariant par déformation des surfaces réelles, la forme de Pontryagin-Viro. Le résultat principal affirme que la composante de l'espace de modules représentée par une surface d'Enriques réelle est déterminée par cette forme combinée avec d'autres invariants topologiques lorsqu'une au moins des composantes de la partie réelle possède une caractéristique d'Euler négative ou nulle. On donne la liste des valeurs de ces invariants.

\section{Version française abrégée}

Une surface d'Enriques réelle est une surface d'Enriques complexe $E$ munie d'une involution antiholomorphe notée conj. On dit que deux surfaces d'Enriques réelles ont le même type de déformation si elles peuvent être reliées par une famille continue à un paramètre de surfaces d'Enriques réelles. Le type topologique de $E_{\mathbb{R}}=$ Fix conj, appelé partie réelle de $E$, est invariant par déformation. On définit un autre invariant de déformation, la décomposition fondamentale de $E_{\mathbb{R}}$ en deux moitiés : deux composantes connexes $C_{a}$ et $C_{b}$ appartiennent à la même moitié si un, et donc tout, lacet de $E$, composé d'un chemin de $C_{a}$ à $C_{b}$ et de son conjugué, est contractible. Ainsi, $E_{\mathbb{R}}$ se scinde en deux $E_{\mathbb{R}}^{(1)}$ et $E_{\mathbb{R}}^{(2)}$, appelées moitiés. La classification à homéomorphisme près des surfaces $E_{\mathbb{R}}$ et de leurs décompositions $\left(E_{\mathbb{R}} ; E_{\mathbb{R}}^{(1)}, E_{\mathbb{R}}^{(2)}\right)$ a été commencée par V. Nikulin [9] et achevée dans [3], [4].

Nous divisons les surfaces d'Enriques réelles en trois groupes : une telle surface est dite de type hyperbolique, parabolique ou elliptique, si la caractéristique d'Euler

This paper was written during the first author's stay at Université Louis Pasteur, Strasbourg, Universitá di Trento, and Université de Rennes I. 
minimale des composantes de $E_{\mathbb{R}}$ est négative, nulle ou positive. Dans cette Note nous présentons la classification à déformation près des surfaces hyperboliques et paraboliques. Elle est obtenue grâce à une version équivariante d'une astuce de Donaldson (basée sur une construction de Hitchin) :

Proposition-clé. La construction équivariante de Donaldson établit une bijection entre les types de déformation des surfaces d'Enriques réelles ayant une moitié marquée non vide et les types de déformation des couples $(\tilde{Y}, \tilde{B})$, où $\tilde{Y}$ est une surface rationnelle réelle et $\tilde{B} \subset \tilde{Y}$ est une courbe non singulière réelle telle que: (1) $\tilde{B} \in\left|-2 K_{\tilde{Y}}\right|$ ( $K$ est la classe canonique), (2) la partie réelle de $\tilde{B}$ est vide et (3) $\tilde{B}$ et la partie réelle $\tilde{Y}_{\mathbb{R}}$ de $\tilde{Y}$ ne sont pas entrelacées (au sens developpé au $§ 1$ ).

Pour classer les surfaces maximales, on introduit au second paragraphe un nouvel invariant appelé forme de Pontrjagin-Viro. Rappelons que l'homomorphisme de Viro envoie le terme $\mathcal{F}^{2} \subset H_{*}\left(E_{\mathbb{R}} ; \mathbb{Z} / 2\right)$ de la filtration de Kalinin dans $H_{2}(E ; \mathbb{Z} / 2)$ (voir [4]). La forme de Pontryagin-Viro, notée $P$, est obtenue en composant cet homomorphisme avec le carré de Pontryagin $\mathcal{P}: H_{2}(E ; \mathbb{Z} / 2) \rightarrow \mathbb{Z} / 4$. L'espace $\mathcal{F}^{2}$ est le sous-espace de $H_{*}\left(E_{\mathbb{R}} ; \mathbb{Z} / 2\right)$ engendré par les classes fondamentales des composantes de $E_{\mathbb{R}}$ et les classes inhomogènes de la forme $\alpha+\left\langle C_{a}-C_{b}\right\rangle$. Ici $\alpha \in H_{1}\left(E_{\mathbb{R}} ; \mathbb{Z} / 2\right)$ et $\left\langle C_{a}-C_{b}\right\rangle \in H_{0}\left(E_{\mathbb{R}} ; \mathbb{Z} / 2\right)$ est la classe réalisée par deux composantes $C_{a}$ et $C_{b}$ sujettes à la condition : ou bien $\alpha^{2}=0$ et $C_{a}, C_{b}$ appartiennent à la même moitié de $E_{\mathbb{R}}$, ou bien $\alpha^{2}=1$ et $C_{a}, C_{b}$ appartiennent aux moitiés différentes. On définit la séparation complexe de $E_{\mathbb{R}}^{(i)}, i=1$ ou 2 , en deux quarts en disant que deux composantes $C_{a}, C_{b}$ sont dans le même quart si $P\left\langle C_{a}-C_{b}\right\rangle=0$. On note $E_{\mathbb{R}}^{(i)}=(1$ er quart $) \sqcup(2$ ème quart $)$.

Une simplification de Morse (topologique) de la partie réelle d'une surface d'Enriques réelle $E$ est une chirurgie élémentaire qui réduit le nombre $\beta_{*}\left(E_{\mathbb{R}}\right)$. Une simplification de Morse est dite algébrique si elle se produit dans une famille continue de surfaces d'Enriques réelles. Nous posons $S_{g}=\#_{g}\left(S^{1} \times S^{1}\right)$ et $V_{q}=\#_{q} \mathbb{R p}^{2}$.

Résultat principal. Toute surface d'Enriques réelle de type hyperbolique ou parabolique est obtenue par une suite de simplifications de Morse algébriques à partir d'une des surfaces extrémales dont la liste est donnée dans les Tableaux 1, 2 et 3. Le type de déformation d'une telle surface est déterminé par la topologie de ses moitiés à trois exceptions suivantes près :

(1) $E_{\mathbb{R}}=V_{10}$ : il y a deux types de déformation; pour l'un des deux types, $E /$ conj est Spin, pour l'autre pas;

(2) $E_{\mathbb{R}}=2 V_{2} \sqcup 4 S$ ou $E_{\mathbb{R}}=V_{2} \sqcup 2 V_{1} \sqcup 3 S$ : leurs types de déformation sont distingués par leur forme de Pontrjagin-Viro; celle-ci est déterminée par la séparation complexe et la valeur $p$ de $P$ sur la classe caractéristique des composantes $V_{2}$; les valeurs possibles sont décrites dans le Tableau 2;

(3) $E_{\mathbb{R}}^{(2)}=4 S$ : chaque type topologique correspond à deux types de déformation distingués par la valeur-nulle ou non-de $\left[E_{\mathbb{R}}^{(2)}\right]$ dans $H_{2}\left(X^{\prime} ; \mathbb{Z} / 2\right)$, où $X^{\prime}$ est le revêtement double de $E /$ conj ramifié le long de $E_{\mathbb{R}}^{(2)}$.

Réciproquement, tout couple $E_{\mathbb{R}}^{(1)} \sqcup E_{\mathbb{R}}^{(2)}$ obtenu par une suite de simplifications de Morse topologiques à partir des couples présentés dans les Tableaux 1-3 peut être réalisé par une surface d'Enriques réelle, à l'exception de deux familles : \{..\} $\sqcup$ $\{5 S\}$ et $\{3 S \sqcup \ldots\} \sqcup\{2 S \sqcup \ldots\}$. 


\section{Introduction}

A real Enriques surface is a complex Enriques surface $E$ equipped with an antiholomorphic involution conj, called complex conjugation; the fixed point set $E_{\mathbb{R}}=$ Fix conj is called the real part of the surface. As is known, the study of a real Enriques surface is equivalent to the study of a real $K 3$-surface $X$ (the double covering of $E$ ) equipped with a fixed point free holomorphic involution $\tau$ (called the Enriques involution) commuting with the real structure (see, e.g., [3]).

Two real Enriques surfaces are said to have the same deformation type if they can be included into a continuous one-parameter family of real Enriques surfaces, or, equivalently, if they belong to the same connected component of the moduli space of real Enriques surfaces. Clearly, the topological type of the real part of a surface is preserved under deformation. (The real part is a closed 2-manifold with finitely many components, each component being either $S_{g}=\#_{g}\left(S^{1} \times S^{1}\right)$ or $V_{q}=\#_{q} \mathbb{R}^{2}$.) Another immediate deformation invariant, which we suggest to call the sign decomposition, comes from the covering $K 3$-surface $X$ : since conj lifts to two real structures $t^{(1)}, t^{(2)}$ on $X$ whose real parts $X_{\mathbb{R}}^{(1)}, X_{\mathbb{R}}^{(2)}$ are disjoint, $E_{\mathbb{R}}$ naturally splits into disjoint union of two halves $E_{\mathbb{R}}^{(i)}=X_{\mathbb{R}}^{(i)} / \tau$, each half being a union of whole components of $E_{\mathbb{R}}$.

The classification of the real parts $E_{\mathbb{R}}$ and decompositions $\left(E_{\mathbb{R}} ; E_{\mathbb{R}}^{(1)}, E_{\mathbb{R}}^{(2)}\right)$ up to homeomorphism was started by V. Nikulin [9] and completed by us in [3], [4]. For most topological types the decomposition $E_{\mathbb{R}}^{(1)} \sqcup E_{\mathbb{R}}^{(2)}$ is subject to the only restriction that the orientation double covering of each half must be the real part of a real $K 3$-surface. However, for the topological types $E_{\mathbb{R}}=k S$ and $E_{\mathbb{R}}=V_{2 q} \sqcup k S$ there are additional congruence type prohibitions, see [4].

It turns natural to divide the real Enriques surfaces in three groups: we say that a real Enriques surface $E$ is of hyperbolic, parabolic, or elliptic type if the minimal Euler characteristic of the components of $E_{\mathbb{R}}$ is negative, zero, or positive, respectively. In this paper we classify up to deformation equivalence the surfaces of hyperbolic and parabolic types. In addition to the sign decomposition and its homological properties (i.e., whether the fundamental classes $\left[E_{\mathbb{R}}\right]$ and/or $\left[E_{\mathbb{R}}^{(i)}\right]$ vanish in $E$ or some auxiliary manifolds) a new invariant, so called Pontrjagin-Viro form (see $\S 2$ ) is necessary (and sufficient) to distinguish the $M$-surfaces, i.e., those with the maximal total $\mathbb{Z} / 2$-Betti number $\beta_{*}\left(E_{\mathbb{R}}\right)=16$. The precise statement is found in $\S 3$. (The information currently available suggests a conjecture that a similar statement is also valid for the surfaces of elliptic type.)

In fact, the approach which we use gives as well some more or less explicit geometrical models of real Enriques surfaces and identifies the components of their moduli space with those of some other, more classical and easier to handle, objects (such as real plane quartics or order 6 curves on quadrics in $P^{3}$ with conditions on the singularities). It is based on an equivariant version of a trick by Donaldson [2], which transforms a real Enriques surface to a real rational surface with a certain curve on it (see $\S 1$; the original construction of [2] is applied to real $K 3$-surfaces).

We are grateful to S. Finashin whose question attracted our attention to the Donaldson construction for real $K 3$-surfaces. 


\section{Donaldson's trick}

When a $K 3$-surface $X$ is equipped with a Kähler metric, it possesses a canonical quaternionic Kähler structure (see [5], where this result is deduced from the Calabi conjecture). Moreover, if $\Omega$ is the fundamental Kähler form and $\Re \omega$ and $\Im \omega$ are, respectively, the real and imaginary parts of a holomorphic 2-form $\omega$, then any nonzero $\mathbb{R}$-linear combination of $\Omega, \Re \omega$, and $\Im \omega$ defines a complex structure on $X$. We normalize $\Omega$ by $\Omega^{2}=(\Im \omega)^{2}\left(=(\Re \omega)^{2}\right)$ and consider the complex structure defined by $\Re \omega$ : it is given by $\varsigma=\Omega-\sqrt{-1} \Im \omega$. The surface $X$ equipped with this new structure will be denoted by $\tilde{X}$.

Let $c$ be a real structure on $X$. Then both the Kähler metric and holomorphic form $\omega$ can be chosen real (the latter in the sense that $c^{*} \omega=\bar{\omega}$ ), and under this condition $c^{*} \varsigma=-\varsigma$, i.e., $c$ is a holomorphic involution on $\tilde{X}$ and its fixed point set is a holomorphic curve in $\tilde{X}$. If, further, $\tau$ is an Enriques involution on $X$ commuting with $c$ (i.e., $c$ is one of the two lifts $t^{(1)}, t^{(2)}$ of a real structure on $\left.X / \tau\right)$ and the Kähler metric is $\tau$-equivariant, then $\tau^{*} \varsigma=\bar{\varsigma}$ (as necessary $\tau^{*} \omega=-\omega$ ); hence, $\tau$ is a real structure on $\tilde{X}$.

In what follows we always assume that $E_{\mathbb{R}}^{(1)} \neq \varnothing$ and $c=t^{(1)}$. Under this assumption the quotient $\tilde{Y}=\tilde{X} / t^{(1)}$ is a real rational surface (the real structure being induced by $\tau$ or $t^{(2)}$ ) and the projection $\tilde{X} \rightarrow \tilde{Y}$ is a real double covering ramified over a nonsingular real curve $\tilde{B}=\operatorname{Fix} t^{(1)} \subset \tilde{Y}$. Clearly, $E_{\mathbb{R}}^{(2)}=\tilde{Y}_{\mathbb{R}}$ and $E_{\mathbb{R}}^{(1)}=\tilde{B} / t^{(1)}$. We say that a real curve $\tilde{B} \subset \tilde{Y}$ with $\tilde{B}_{\mathbb{R}}=\varnothing$ is not linked with $\tilde{Y}_{\mathbb{R}}$ if for any path $\gamma:[0,1] \rightarrow \tilde{Y} \backslash \tilde{B}$ with $\gamma(0), \gamma(1) \in \tilde{Y}_{\mathbb{R}}$ the loop $\gamma^{-1} \cdot \operatorname{conj} \tilde{Y} \gamma$ is $\mathbb{Z} / 2$-homologous to zero in $\tilde{Y} \backslash \tilde{B}$.

1.1. Theorem. The Donaldson construction establishes a one-to-one correspondence between the deformation classes of real Enriques surfaces with distinguished nonempty half (i.e., pairs $\left(E, E_{\mathbb{R}}^{(1)}\right)$ with $E_{\mathbb{R}}^{(1)} \neq \varnothing$ ) and deformation classes of pairs $(\tilde{Y}, \tilde{B})$, where $\tilde{Y}$ is a real rational surface and $\tilde{B} \subset \tilde{Y}$ is a nonsingular real curve such that that (1) $\tilde{B} \in\left|-2 K_{\tilde{Y}}\right|$ ( $K$ being the canonical class), (2) the real point set of $\tilde{B}$ is empty, and (3) $\tilde{B}$ is not linked with the real point set $\tilde{Y}_{\mathbb{R}}$ of $\tilde{Y}$.

(In this statement the first condition on $\tilde{B}$ ensures that the double covering $\tilde{X}$ of $\tilde{Y}$ ramified along $\tilde{B}$ is a $K 3$-surface; the two others are equivalent to the requirement that one of the two lifts of the real structure to $\tilde{X}$ have empty fixed point set.)

Remark. Note that the fact that for $K 3$-surfaces the classification of real structures coincides with that of holomorphic involutions reversing $\omega$ was first observed by V. Nikulin in [7] (without a geometrical explanation of this phenomenon; the two problems just lead to the same arithmetical question). In [7], [8] Nikulin also started the investigation of the resulting rational surfaces, which generalize to an extent the notion of Del-Pezzo surface. In particular, he showed that the minimal model of such a surface is either $\mathbb{C} P^{2}$ or the scroll $F_{n}$ with $n=0,2,3$, or 4 .

Our investigation of real Enriques surfaces is based on Theorem 1.1 and the following result, mainly due to A. Comessatti (see [6]):

1.2. Theorem. The following is the complete list of minimal over $\mathbb{R}$ real rational surfaces: (1) $\mathbb{C} P^{2}$ with the standard real structure; (2) the four real structures on $\mathbb{C} P^{1} \times \mathbb{C} P^{1} ;(3)$ the scrolls $F_{n}$ with $n \geqslant 2$ (there are two real structures for $n$ even 
and one real structure for $n$ odd); (4) conic bundles with $2 n \geqslant 8$ singular fibers which all consist of pairs of conjugate imaginary lines; (5) Del-Pezzo surfaces of degree 1 with the real point set $V_{1} \sqcup 4 S$; (6) Del-Pezzo surfaces of degree 2 with the real point set $3 S$ or $4 S$.

In order to study the real structures of pairs $(\tilde{Y}, \tilde{B})$ we use the anti-canonical (or anti-bicanonical) model of $\tilde{Y}$ (which must certainly preserve the real structure). If $\tilde{B}$ has at least one component of positive genus (it is this case that is presently covered by our results), such a model exists, possibly, after several real blow-downs which can still be controlled.

\section{Pontrjagin-Viro form on $M$-surfaces}

Let $E$ be an $M$-surface. Then its Kalinin's spectral sequence degenerates; hence, the Viro homomorphism $b_{2}$ maps the middle term $\mathcal{F}^{2}$ of Kalinin's filtration onto $H_{2}(E ; \mathbb{Z} / 2)$, and one can combine it with the Pontrjagin square $\mathcal{P}: H_{2}(E ; \mathbb{Z} / 2) \rightarrow$ $\mathbb{Z} / 4$ and define a quadratic refinement $P=\mathcal{P} \circ$ bv $_{2}: \mathcal{F}^{2} \rightarrow \mathbb{Z} / 4$ of Kalinin's intersection form. (Details on Kalinin's spectral sequence and Viro homomorphisms can be found, e.g., in [4]. Recall that the Pontrjagin square of a class $y \in H_{2}(E ; \mathbb{Z} / 2)$ realized by an embedded surface $F$ equals $[F] \circ[F]+2 \chi(F) \bmod 4$, where $[F] \circ[F]$ is the normal Euler number of $F$.)

If $E$ is an Enriques surface, $\mathcal{F}^{2}$ is spanned by the fundamental classes $\left[C_{i}\right]$ of the components of $E_{\mathbb{R}}$ and classes of the form $\alpha+\left\langle C_{a}-C_{b}\right\rangle$, where $\alpha \in H_{1}\left(E_{\mathbb{R}} ; \mathbb{Z} / 2\right)$, $\left\langle C_{a}-C_{b}\right\rangle$ is the 0-dimensional class realized by two components $C_{a}, C_{b}$, and either $\alpha^{2}=0$ and $C_{a}, C_{b}$ belong to the same half of $E_{\mathbb{R}}$, or $\alpha^{2}=1$ and $C_{a}, C_{b}$ belong to distinct halves. The restriction of $P$ to $\left\{\sum\left\langle C_{a}-\mathbb{C}_{b}\right\rangle \mid C_{a}, C_{b} \in E_{\mathbb{R}}^{(i)}\right\}$ is a homomorphism (with values in $\mathbb{Z} / 2 \stackrel{\cdot 2}{\longrightarrow} \mathbb{Z} / 4$ ); hence, it divides $E_{\mathbb{R}}^{(i)}$ into two 'quarters': two components $C_{a}, C_{b}$ belong to the same quarter iff $P\left\langle C_{a}-C_{b}\right\rangle=0$. Following G. Mikhalkin we call this subdivision the complex separation of $E_{\mathbb{R}}$. To indicate the splitting of a half into quarters we use the notation $E_{\mathbb{R}}^{(i)}=$ (quarter 1) $\sqcup$ (quarter 2).

Generalizations of this construction and its relation to other structures known in topology of real algebraic surfaces (such as Mikhalkin's complex separation, Guillou-Marin-Rokhlin form on characteristic surfaces in the quotient $E /$ conj, and quadratic forms constructed by Viro) are discussed in [1].

\section{Main result}

A (topological) Morse simplification of the real part of a real Enriques surface $E$ is a Morse surgery reducing the total $\mathbb{Z} / 2$-Betti number $\beta_{*}\left(E_{\mathbb{R}}\right)$. A simplification is called algebraic if it may occur in a continuous family of real Enriques surfaces. A surface $E$ (or a half $E_{\mathbb{R}}^{(i)}$ ) is said to be of type $\mathrm{I}$ if $\left[E_{\mathbb{R}}\right]$ (respectively, $\left[E_{\mathbb{R}}^{(i)}\right]$ ) equals 0 or $w_{2}(E)$ in $H_{2}(E ; \mathbb{Z} / 2)$.

3.1. Main Theorem. Any real Enriques surface of hyperbolic or parabolic type is obtained by a series of algebraic Morse simplifications from one of the extremal surfaces (which are all of type I) listed in Tables 1, 2, and 3 (where $a^{*}$ indicates that both the halves are of type I.) With few exceptions the deformation type of a surface is determined by its sign decomposition. The exceptions are:

(1) surfaces with $E_{\mathbb{R}}=V_{10}$ : there are two deformation types which differ by 
whether $E /$ conj is Spin or not;

(2) $M$-surfaces of parabolic type with $E_{\mathbb{R}}=2 V_{2} \sqcup 4 S$ or $E_{\mathbb{R}}=V_{2} \sqcup 2 V_{1} \sqcup 3 S$ : the deformation types are distinguished by the Pontrjagin-Viro form; the latter can be recovered from the complex separation and the value $p=P(\alpha)$ on the characteristic class of the components $V_{2}$, see Table 2 (if there are two such components, the values of $P(\alpha)$ on them coincide);

(3) surfaces of parabolic type with $E_{\mathbb{R}}^{(2)}=4 S$ : each topological type corresponds to two deformation types which differ by whether $\left[E_{\mathbb{R}}^{(2)}\right]$ vanishes in $H_{2}\left(X / t^{(1)} ; \mathbb{Z} / 2\right)$ or not.

Conversely, with the exception of $\{\ldots\} \sqcup\{5 S\}$ and $\{3 S \sqcup \ldots\} \sqcup\{2 S \sqcup \ldots\}$ any pair $E_{\mathbb{R}}^{(1)} \sqcup E_{\mathbb{R}}^{(2)}$ obtained by a series of topological Morse simplifications from one of those listed in Tables 1-3 can be realized as the real part of a real Enriques surface.

Remark. Note that there is another approach to the moduli problem (see [10]), more traditional for objects related to $K 3$-surfaces. Namely, the assignment conj $\mapsto$ $\left(t_{*}^{(1)}, t_{*}^{(2)}\right)$ establishes a one-to-one correspondence between the deformation classes of real Enriques surfaces and isomorphism classes of commuting pairs of involutions on the lattice $H_{2}(X) \cong E_{8} \oplus E_{8} \oplus U \oplus U \oplus U$ such that the eigenspaces $L_{\epsilon^{(1)}, \epsilon^{(2)}}=$ $\left\{x \in L \mid t_{*}^{(i)} x=\epsilon^{(i)} x\right\}$ have the inertia indices $\sigma_{+} L_{++}=0, \sigma_{+} L_{+-}=\sigma_{+} L_{-+}=$ $\sigma_{+} L_{--}=1$ and the eigenlattice $\left\{x \in L \mid t_{*}^{(1)} t_{*}^{(2)} x=x\right\}$ is the double of $E_{8} \oplus U$.

This approach covers all real Enriques surfaces. However, it is not evident how to enumerate the actions (whose number is greater than 400) and how to recover the topological invariants (and even the topological type) of the real part from the arithmetical properties of the action and vice versa.

\section{References}

1. A. Degtyarev, On the Pontrjagin-Viro form on a real algebraic surface (to appear).

2. S. Donaldson, Yang-Mills invariants of smooth four-manifolds, Geometry of Low Dimensional Manifolds, Cambridge Univ. Press, Cambridge, vol. 1, 1990, pp. 5-40.

3. A. Degtyarev, V. Kharlamov, Topological classification of real Enriques surfaces, Topology 35 (1996), no. 3, 711-729.

4. A. Degtyarev, V. Kharlamov, Halves of a Real Enriques Surface, to appear in Comm. Math. Helv.; Current version: Distribution of the components of a real Enriques surface, Preprint of the Max-Planck Institute, MPI/95-58, 1995.

5. N. Hitchin, Compact four-dimensional Einstein manifolds, J. Diff. Geom. 9 (1974), 435-441.

6. V. A. Iskovskikh, Minimal models of rational surfaces over arbitrary fields, Izv. Akad. Nauk SSSR Ser. Mat. 43 (1979), 19-43 (Russian); English trans. in Math. USSR-Izv. 14 (1980), $17-39$.

7. V. V. Nikulin, On the quotient groups of the automorphisme groups of hyperbolic forms by the subgroups generated by 2-reflections. Algebraic-geometric applications, Contemporary Problems in Mathematics (VINITI Moscow), vol. 18, 1981, pp. 3-114 (Russian); English trans. in J. Soviet Math. vol. 22, 1983, pp. 1401-1476.

8. V. V. Nikulin, Discrete Reflection Groups in Lobachevsky Spaces and Algebraic Surfaces, Proceedings of ICM, Berkley, 1986, pp. 654-671.

9. V. V. Nikulin, On the topological classification of real Enriques surfaces. I, Amer. Math. Soc. Transl. (2) 173 (1996), 187-202.

10. V. V. Nikulin, Involutions of integral quadratic forms and their application to real algebraic geometry, Izv. Akad. Nauk SSSR Ser. Mat. 47 (1983), 109-188 (Russian); English trans. in Math. USSR-Izv. 22 (1984), 99-172. 
TABLE 1

Extremal hyperbolic types

\begin{tabular}{lllll}
$\frac{\beta_{*}=16, \chi=-8}{*}$ & \multicolumn{1}{c}{$\beta_{*}=16, \chi=8$} & & $\beta_{*}=12, \chi=0$ \\
$*\left\{V_{11} \sqcup V_{1}\right\} \sqcup\{\varnothing\}$ & $*\left\{V_{4} \sqcup S\right\} \sqcup\{4 S\}$ & & $\left\{V_{6}\right\} \sqcup\{2 S\}$ & $*\left\{V_{4} \sqcup S\right\} \sqcup\left\{S_{1}\right\}$ \\
$*\left\{V_{10}\right\} \sqcup\left\{S_{1}\right\}$ & $*\left\{V_{3} \sqcup V_{1} \sqcup 4 S\right\} \sqcup\{\varnothing\}$ & $*\left\{V_{5} \sqcup V_{1} \sqcup S\right\} \sqcup\{\varnothing\}$ & $\left\{V_{4} \sqcup S\right\} \sqcup\left\{V_{2}\right\}$ \\
$\left\{V_{11}\right\} \sqcup\left\{V_{1}\right\}$ & $\left\{V_{3} \sqcup V_{1} \sqcup 3 S\right\} \sqcup\{S\}$ & & $\left\{V_{5} \sqcup V_{1}\right\} \sqcup\{S\}$ & $\left\{V_{4}\right\} \sqcup\left\{V_{2} \sqcup S\right\}$ \\
$\left\{V_{10}\right\} \sqcup\left\{V_{2}\right\}$ & $\left\{V_{3} \sqcup V_{1} \sqcup 2 S\right\} \sqcup\{2 S\}$ & $\left\{V_{5} \sqcup S\right\} \sqcup\left\{V_{1}\right\}$ & $\left\{V_{3} \sqcup V_{1}\right\} \sqcup\left\{V_{2}\right\}$ \\
$\left\{V_{9}\right\} \sqcup\left\{V_{3}\right\}$ & $\left\{V_{3} \sqcup V_{1} \sqcup S\right\} \sqcup\{3 S\}$ & $\left\{V_{5}\right\} \sqcup\left\{V_{1} \sqcup S\right\}$ & $\left\{V_{3}\right\} \sqcup\left\{V_{2} \sqcup V_{1}\right\}$ \\
$\left\{V_{8}\right\} \sqcup\left\{V_{4}\right\}$ & $\left\{V_{3} \sqcup V_{1}\right\} \sqcup\{4 S\}$ & & $*\left\{V_{4} \sqcup 2 V_{1}\right\} \sqcup\{\varnothing\}$ & $\left\{V_{3} \sqcup S\right\} \sqcup\left\{V_{3}\right\}$ \\
$\left\{V_{7}\right\} \sqcup\left\{V_{5}\right\}$ & $\left\{V_{3} \sqcup 4 S\right\} \sqcup\left\{V_{1}\right\}$ & & $\left\{V_{4} \sqcup V_{1}\right\} \sqcup\left\{V_{1}\right\}$ & \\
$\left\{V_{6}\right\} \sqcup\left\{V_{6}\right\}$ & $\left\{V_{3} \sqcup 3 S\right\} \sqcup\left\{V_{1} \sqcup S\right\}$ & & $\left\{V_{4}\right\} \sqcup\left\{2 V_{1}\right\}$ & \\
& $\left\{V_{3} \sqcup 2 S\right\} \sqcup\left\{V_{1} \sqcup 2 S\right\}$ & & \\
& $\left\{V_{3} \sqcup S\right\} \sqcup\left\{V_{1} \sqcup 3 S\right\}$ & & \\
& $\left\{V_{3}\right\} \sqcup\left\{V_{1} \sqcup 4 S\right\}$ & &
\end{tabular}

TABLE 2

Extremal parabolic types with $\beta_{*}=16, \chi=8$

\begin{tabular}{|c|c|c|c|c|c|}
\hline$E_{\mathbb{R}}^{(1)}$ & $E_{\mathbb{R}}^{(2)}$ & $p$ & $E_{\mathbb{R}}^{(1)}$ & $E_{\mathbb{R}}^{(2)}$ & $p$ \\
\hline \multicolumn{3}{|c|}{ Case $E_{\mathbb{R}}=S_{1} \sqcup V_{2} \sqcup 4 S$} & \multicolumn{3}{|c|}{ Case $E_{\mathbb{R}}=V_{2} \sqcup 2 V_{1} \sqcup 3 S$ (continued) } \\
\hline$*\left(V_{2} \sqcup 2 S\right) \sqcup(2 S)$ & $\left(S_{1}\right) \sqcup(\varnothing)$ & 0 & $\left(V_{2} \sqcup S\right) \sqcup\left(V_{1} \sqcup S\right)$ & $\left(V_{1}\right) \sqcup(S)$ & 0 \\
\hline \multicolumn{3}{|c|}{ Case $E_{\mathbb{R}}=2 V_{2} \sqcup 4 S$} & $\begin{array}{l}\left(V_{2} \sqcup S\right) \sqcup\left(V_{1} \sqcup S\right) \\
\left(V_{2} \sqcup V_{1} \sqcup S\right) \sqcup(S)\end{array}$ & $\begin{array}{l}\left(V_{1} \sqcup S\right) \sqcup(\varnothing) \\
\left(V_{1}\right) \sqcup(S)\end{array}$ & $\begin{array}{c}2 \\
0 \text { or } 2\end{array}$ \\
\hline $\begin{array}{l}*\left(V_{2}\right) \sqcup\left(V_{2}\right) \\
*\left(V_{2}\right) \sqcup\left(V_{2}\right) \\
*\left(2 V_{2}\right) \sqcup(\varnothing)\end{array}$ & $\begin{array}{l}(2 S) \sqcup(2 S) \\
(3 S) \sqcup(S) \\
(2 S) \sqcup(2 S)\end{array}$ & $\begin{array}{c}0 \\
2 \\
0 \text { or } 2\end{array}$ & $\begin{array}{l}\left(V_{2} \sqcup S\right) \sqcup\left(V_{1}\right) \\
\left(V_{2} \sqcup S\right) \sqcup\left(V_{1}\right) \\
\left(V_{2} \sqcup V_{1}\right) \sqcup(S)\end{array}$ & $\begin{array}{l}\left(V_{1} \sqcup S\right) \sqcup(S) \\
\left(V_{1}\right) \sqcup(2 S) \\
\left(V_{1} \sqcup S\right) \sqcup(S)\end{array}$ & $\begin{array}{c}0 \\
2 \\
0 \text { or } 2\end{array}$ \\
\hline$\left(V_{2} \sqcup 2 S\right) \sqcup(2 S)$ & $\left(V_{2}\right) \sqcup(\varnothing)$ & 0 & $\left(V_{2}\right) \sqcup\left(V_{1}\right)$ & $\left(V_{1} \sqcup S\right) \sqcup(2 S)$ & 0 \\
\hline $\begin{array}{l}\left(V_{2} \sqcup S\right) \sqcup(2 S) \\
\left(V_{2} \sqcup 2 S\right) \sqcup(S)\end{array}$ & $\begin{array}{l}\left(V_{2} \sqcup S\right) \sqcup(\varnothing) \\
\left(V_{2}\right) \sqcup(S)\end{array}$ & $\begin{array}{l}2 \\
2\end{array}$ & $\begin{array}{l}\left(V_{2}\right) \sqcup\left(V_{1}\right) \\
\left(V_{2} \sqcup V_{1}\right) \sqcup(\varnothing)\end{array}$ & $\begin{array}{l}\left(V_{1} \sqcup 2 S\right) \sqcup(S) \\
\left(V_{1} \sqcup S\right) \sqcup(2 S)\end{array}$ & $\begin{array}{c}2 \\
0 \text { or } 2\end{array}$ \\
\hline$\left(V_{2} \sqcup S\right) \sqcup(S)$ & $\left(V_{2} \sqcup S\right) \sqcup(S)$ & 0 & $\left(V_{2} \sqcup S\right) \sqcup(2 S)$ & $\left(V_{1}\right) \sqcup\left(V_{1}\right)$ & 0 \\
\hline \multicolumn{3}{|c|}{ Case $E_{\mathbb{R}}=V_{2} \sqcup 2 V_{1} \sqcup 3 S$} & $\left(V_{2} \sqcup S\right) \sqcup(2 S)$ & $\left(2 V_{1}\right) \sqcup(\varnothing)$ & 2 \\
\hline$*\left(V_{2} \sqcup 2 S\right) \sqcup\left(2 V_{1} \sqcup S\right)$ & $\varnothing$ & 0 & $\left(V_{2} \sqcup 2 S\right) \sqcup(S)$ & $\left(V_{1}\right) \sqcup\left(V_{1}\right)$ & 0 \\
\hline * $\left(V_{2} \sqcup 2 V_{1} \sqcup S\right) \sqcup(2 S)$ & $\varnothing$ & 0 or 2 & $\left(V_{2} \sqcup S\right) \sqcup(S)$ & $\left(2 V_{1}\right) \sqcup(S)$ & 0 \\
\hline$\left(V_{2} \sqcup V_{1} \sqcup S\right) \sqcup\left(V_{1} \sqcup S\right)$ & $(S) \sqcup(\varnothing)$ & 0 or 2 & $\left(V_{2} \sqcup S\right) \sqcup(S)$ & $\left(V_{1} \sqcup S\right) \sqcup\left(V_{1}\right)$ & 2 \\
\hline $\begin{array}{l}\left(V_{2} \sqcup S\right) \sqcup\left(2 V_{1}\right) \\
\left(V_{2} \sqcup S\right) \sqcup\left(2 V_{1}\right) \\
\left(V_{2} \sqcup 2 V_{1}\right) \sqcup(S)\end{array}$ & $\begin{array}{l}(S) \sqcup(S) \\
(2 S) \sqcup(\varnothing) \\
(S) \sqcup(S)\end{array}$ & $\begin{array}{c}0 \\
2 \\
0 \text { or } 2\end{array}$ & $\begin{array}{l}\left(V_{2}\right) \sqcup(S) \\
\left(V_{2}\right) \sqcup(S) \\
\left(V_{2} \sqcup S\right) \sqcup(\varnothing) \\
\left(V_{2} \sqcup S\right) \sqcup(\varnothing)\end{array}$ & $\begin{array}{l}\left(V_{1} \sqcup S\right) \sqcup\left(V_{1} \sqcup S\right) \\
\left(2 V_{1} \sqcup S\right) \sqcup(S) \\
\left(V_{1} \sqcup S\right) \sqcup\left(V_{1} \sqcup S\right) \\
\left(2 V_{1}\right) \sqcup(2 S)\end{array}$ & $\begin{array}{l}0 \\
2 \\
0 \\
2\end{array}$ \\
\hline$\left(V_{2} \sqcup V_{1}\right) \sqcup\left(V_{1}\right)$ & $(2 S) \sqcup(S)$ & 0 or 2 & $\left(V_{2}\right) \sqcup(\varnothing)$ & $\left(2 V_{1} \sqcup S\right) \sqcup(2 S)$ & 0 \\
\hline $\begin{array}{l}\left(V_{2} \sqcup 2 S\right) \sqcup\left(V_{1} \sqcup S\right) \\
\left(V_{2} \sqcup V_{1} \sqcup S\right) \sqcup(2 S)\end{array}$ & $\begin{array}{l}\left(V_{1}\right) \sqcup(\varnothing) \\
\left(V_{1}\right) \sqcup(\varnothing)\end{array}$ & $\begin{array}{c}0 \\
0 \text { or } 2\end{array}$ & $\left(V_{2}\right) \sqcup(\varnothing)$ & $\left(V_{1} \sqcup 2 S\right) \sqcup\left(V_{1} \sqcup S\right)$ & 2 \\
\hline
\end{tabular}

\section{TABLE 3}

Other special parabolic types

Extremal

$*\left\{S_{1}\right\} \sqcup\left\{2 V_{2}\right\} \quad \beta_{*}=12, \chi=0$

$*\left\{S_{1}\right\} \sqcup\left\{S_{1}\right\} \quad \beta_{*}=8, \quad \chi=0$

$*\left\{S_{1}\right\} \sqcup\{4 S\} \quad \beta_{*}=12, \chi=8 \quad 2$ types
Nonextremal

$\left\{V_{2} \sqcup S\right\} \sqcup\{4 S\} \quad \beta_{*}=14, \chi=10 \quad 2$ types $\left\{V_{2}\right\} \sqcup\{4 S\} \quad \beta_{*}=12, \chi=8 \quad 2$ types 